\title{
Short-Term Impact of Iron Deficiency in Different Subsets of Patients with Precapillary Pulmonary Hypertension from an Eastern European Pulmonary Hypertension Referral Center
}

Ioan Tilea (iD)

Dorina Nastasia Petra

Razvan Constantin Serban

Manuela Rozalia Gabor

Mariana Cornelia Tilinca

Leonard Azamfirei iD

Andreea Varga (iD

'Department of Internal Medicine, "George Emil Palade" University of Medicine,

Pharmacy, Science and Technology of Targu Mures, Targu Mures, 540I42, Romania;

${ }^{2}$ Department of Cardiology II, County Emergency Clinical Hospital, Targu Mures, 540042, Romania; ${ }^{3}$ Department of Family Medicine, "George Emil Palade" University of Medicine, Pharmacy, Science and Technology of Targu Mures, Targu Mures, 540 I42, Romania; ${ }^{4}$ Department of Internal Medicine II, County Emergency Clinical Hospital, Targu Mures, 540042, Romania; ${ }^{5}$ Cardiac Catheterization Laboratory, The Emergency Institute for Cardiovascular Diseases and Transplantation, Targu Mures, 540136, Romania; ${ }^{6}$ Department of Economics and Law, "George Emil Palade" University of Medicine, Pharmacy, Science and Technology of Targu Mures, Targu Mures, 540142, Romania; ${ }^{7}$ Department of Anesthesiology and Intensive Care, "George Emil Palade" University of Medicine, Pharmacy, Science and Technology of Targu Mures, Targu Mures, 540I42, Romania; ${ }^{8}$ Department of Anesthesiology and Intensive Care, County Emergency Clinical Hospital, Targu Mures, 540042, Romania

Correspondence: Dorina Nastasia Petra Department of Family Medicine, "George Emil Palade" University of Medicine,

Pharmacy, Science and Technology of

Targu Mures, Targu Mures, 540142,

Romania

Tel +40740194122

Email dorina.petra@umfst.ro
Background: Over the last few decades, interest in the role of iron status in pulmonary hypertension $(\mathrm{PH})$ has grown considerably due to its potential impact on symptoms, exercise capacity (as assessed by the 6-minute walk distance [6MWD]), prognosis, and mortality. The aim of the present study was to identify iron deficiency (ID) prevalence in specific precapillary PH subgroups of Romanian patients and its short-term impact on 6MWD.

Patients and Methods: Complete datasets from 25 precapillary $\mathrm{PH}$ adults were examined and included in the analysis. Data were collected at baseline and after continuous follow-up of an average of 13.5 months. Enrolled patients were assigned to group 1 (pulmonary arterial hypertension) or subgroup 4.1 (chronic thromboembolic pulmonary hypertension), and individualized targeted therapy was prescribed. General characteristics, World Health Organization functional class, 6MWD, pulse oximetry, laboratory parameters, and echocardiographic and hemodynamic parameters were recorded. Ferritin values and transferrin saturation were used to assess ID.

Results: At baseline, 16 out of 25 patients were iron deficient. The univariate linear regression analysis did not show a statistically significant impact of ID on $6 \mathrm{MWD}(\mathrm{p}=0.428)$. In multivariate regression analysis, possible predictors of $6 \mathrm{MWD}$, including ID, were not statistically significant at baseline or after an average of 13.5 months follow-up ( $\mathrm{p}=0.438,0.361$, respectively) and ID indicates a negative impact on 6MWD independent of applied corrections.

Conclusion: The results of this study demonstrate that 1.4.1 subgroup PAH patients have an increased prevalence of ID compared with other etiologies. ID has a negative impact on the functional status (assessed by 6MWD), in specific groups and subgroups of patients with precapillary $\mathrm{PH}$, albeit not independently nor significant to other known predictors such as age, gender, oxygen saturation, and hemoglobin value. These data can be integrated with global research and are consistent with phenotypes of patients diagnosed with $\mathrm{PH}$ of different etiologies.

Keywords: pulmonary arterial hypertension, chronic thromboembolic pulmonary hypertension, ferritin, iron replenishment, six-minute walk distance

\section{Introduction}

According to current guidelines, pulmonary hypertension ( $\mathrm{PH})$ is defined as an increase in mean pulmonary arterial pressure (mPAP) to $\geq 25 \mathrm{mmHg}$ at rest as determined by right heart catheterization. ${ }^{1,2}$ An $\mathrm{mPAP}$ of $\geq 25 \mathrm{mmHg}$ is associated with a pulmonary artery wedge pressure (PWP) of $\leq 15 \mathrm{mmHg}$, whereas a pulmonary vascular resistance (PVR) of $\geq 3.0$ Wood units defines precapillary $\mathrm{PH}^{1}$ An updated hemodynamic definition of 
pulmonary hypertension was released in 2019 at the Sixth World Symposium on Pulmonary Hypertension. The proposed new definition is characterized by $\mathrm{mPAP}>20 \mathrm{mmHg}$, while precapillary $\mathrm{PH}$ is defined as $\mathrm{mPAP}>20 \mathrm{mmHg}$, $\mathrm{PWP} \leq 15$ mmHg and PVR $\geq 3 \mathrm{WU}^{2}$ The hemodynamic definition takes both pathological factors and therapeutic approaches into account to classify the clinical conditions associated with $\mathrm{PH}$ in five patient groups. ${ }^{1}$

Worldwide, the incidence of PH is not systematically reported, nor is data related to the prevalence of pulmonary arterial hypertension (PAH, group 1) and chronic thromboembolic pulmonary hypertension (CTEPH, subgroup 4.1) in different subgroups. Both PAH and CTEPH are rare and devastating diseases, which invariably progress to right heart failure and premature death. ${ }^{3}$ Documentation of $\mathrm{PH}$ epidemiology is necessary for standardization of diagnostic and therapeutic management practices, with a particular emphasis on aligning variations among different geographic and economic regions worldwide. Details of the incidence, prevalence, and survival rate of PAH and CTEPH patients in Central and Eastern Europe are published in the Czech Republic registry, Russian National Registry, and Latvian nationwide $\mathrm{PH}$ registry. ${ }^{4-6}$

Several risk assessment tools have been developed to stratify patients, assess mortality risk, and tailor therapy in pulmonary hypertension cases. ${ }^{1,7-10}$ Sonnweber et al published a comparative analysis in which seven different risk assessment methods were compared, and concluded that there is still a need for new tools in intermediate- and high-risk precapillary $\mathrm{PH}$ patients, the present methods being inaccurate for predicting mortality. ${ }^{11}$

In some clinical circumstances, modified, simplified score calculators that include little accessible data and undependable or invasive hemodynamic parameters can be used to predict 1-year mortality and long-term transplant-free survival in adult $\mathrm{PAH}$ or $\mathrm{PAH}$ associated with congenital heart disease (PAH-CHD) patients. ${ }^{12-15}$

Over the few past decades, considerable interest in the role of iron status in idiopathic PAH and PAH-CHD has developed due to its potential impact on symptoms, exercise capacity (as assessed by the 6-minute walk distance [6MWD]), prognosis, and mortality. ${ }^{16,17}$ Iron deficiency is highly prevalent in $\mathrm{PH}$ patients, ranging from $27 \%$ to $63 \%$ of cases. ${ }^{18}$ In current practice, decreased values of serum ferritin and transferrin saturation indicate iron deficiency the need for iron replenishment. Several studies have evaluated the potential benefit of iron administration, highlighting significant positive outcomes in terms of improved exercise capacity, New York Heart
Association (NYHA) functional classification of heart failure, and quality of life. ${ }^{19}$ Correction of iron deficiency has to be done with caution, especially in PAH-CHD patients because an excessive erythropoietic response could aggravate blood viscosity, as suggested by Broberg et al. ${ }^{20}$

Available data about the management of PAH and CTEPH patients in Romania are minimal, especially concerning short- and medium-term survival.

This research aimed to identify iron deficiency prevalence, and its short-term impact (determination and prediction) on functional capacity as assessed by the 6MWD test, in specific precapillary pulmonary hypertension groups and subgroups of patients from a single Romanian referral center. This is the first study presenting real-life data in a cluster of Romanian PAH and CTEPH patients over 18 years of age.

\section{Materials and Methods}

\section{Study Participants and Design}

Data records from a total of 48 precapillary $\mathrm{PH}$ patients (October 2015 to February 2021) were examined at the beginning of this retrospective study. At the time of the analysis, the records were incomplete for 23 , and consequently a cohort of 25 adult patients ( 15 women), diagnosed and treated according to recommendations and drugs approved in Romania, were included in the analysis. The mean sample age was $46.40 \pm 18.64$ years. The females were on average younger than the males (40.07 vs 55.94 years).

The present research includes data (demographic, laboratory test, functional status) available for each patient at baseline and continuously thereafter over a mean period of 13.5 months. We regard our study as short-term given this brief follow-up.

The data were obtained from a tertiary-level institution, the County Emergency Clinical Hospital, National Centre for Adult Pulmonary Hypertension Treatment, Targu Mures, Romania.

Depending on the clinical diagnosis, the enrolled patients were assigned to group 1 (PAH) or group 4, subgroup 4.1 (chronic thromboembolic PH-CTEPH). PAH patients were assigned to specific subgroups: 1.1, idiopathic PAH (iPAH); 1.4.1, PAH associated with connective tissue disease (PAH-CTD); or 1.4.4, PAH associated with congenital heart disease (PAH-CHD).

Patients with hematological disorders, active bleeding, ongoing oral iron supplementation, advanced chronic kidney disease with a glomerular filtration rate of less than $30 \mathrm{~mL} /$ 
$\min / 1.73 \mathrm{~m}^{2}$ (CKD-EPI formula), severe liver disease, established endocrine disorders, and changes in $\mathrm{PH}$ therapy within three months prior to screening were excluded.

All patients were on individualized, targeted therapy. Specific drug treatment options at the time of the study were sildenafil, bosentan, macitentan and riociguat. The majority of CTEPH patients were medically treated as pulmonary endarterectomy (PEA) was inaccessible; only for two inoperable patients was balloon pulmonary angioplasty (BPA) offered. Supportive therapies (oral anticoagulants, diuretics, oxygen, and cardiovascular drugs, but excluding iron substitution) were adjusted individually as appropriate to patient status.

\section{Associated Variables Considered in the Study}

At enrollment, associated variables were recorded, including general characteristics (age, gender, body mass index), World Health Organization (WHO) functional class of $\mathrm{PH}$, 6MWD, pulse oximetry, laboratory parameters (including NT-proBNP [N-terminal pro-brain natriuretic peptide]), echocardiographic studies, and hemodynamic parameters. For each patient, blood pressure at rest and electrocardiograms were acquired. Fasting peripheral blood samples were collected from an antecubital vein at baseline and during follow up, and analyzed at a local ISO-15189certified laboratory.

The laboratory parameters included complete blood count, serum iron, creatinine, aspartate aminotransferase, alanine aminotransferase, and uric acid. Serum ferritin and transferrin were determined by immunoturbidimetry. Fibrinogen plasma levels were calculated by coagulometry. Serum NT-proBNP levels were measured using a fluorescence immunoassay.

Iron status was assessed from baseline serum levels of iron (normal range: 9.0-30 $\mu \mathrm{mol} / \mathrm{L}$ ), ferritin (normal range 30-400 $\mu \mathrm{g} / \mathrm{L}$ [male], 30-150 $\mu \mathrm{g} / \mathrm{L}$ [female]), and transferrin (laboratory range: $228-428 \mu \mathrm{g} / \mathrm{dL}$ ). Transferrin saturation (TSAT) was calculated from iron saturation and total iron binding capacity (TIBC) as inferred from transferrin concentration. $^{21,22}$ Iron deficiency was defined using the strict definition proposed by Sonnweber et al. ${ }^{23}$ Anemia was defined as a hemoglobin level of less than $13.0 \mathrm{~g} / \mathrm{L}$ for males and $12.0 \mathrm{~g} / \mathrm{L}$ for females. Iron deficiency status related to common co-morbidities (for example, gastrointestinal diseases, malignancy, hemorrhage, hemosiderinuria, hemoglobinuria) was excluded. In iron-deficiency patients, parenteral replenishment of iron stores was carried out with ferric carboxymaltose or Fe(III)-hydroxide sucrose (Ferinject $\AA$, Venofer ${ }^{\circledR}$, respectively, Vifor Pharma, Switzerland). ${ }^{24}$ The maximum administered dose was $15 \mathrm{mg}$ iron/kg body weight, and the total iron dose was calculated according to the Ganzoni formula using a web application (https://www.mdcalc.com/gan zoni-equation-iron-deficiency-anemia).

Standard ultrasound evaluation of cardiac chambers, valvulopathies and pulmonary circulation was performed (GE Vivid ${ }^{\mathrm{TM}}$ E9 ultrasound system, GE Healthcare, Boston, MA, USA) at enrollment and at regular visits during follow-up. All exams were performed according to current guidelines. ${ }^{25,26}$

Diagnostic and comprehensive hemodynamic assessments were carried out by standard right-heart catheterization (RHC) via the right femoral vein. ${ }^{27} \mathrm{RHC}$ was provided in the Emergency Institute for Cardiovascular Diseases and Transplantation of Targu Mures, Romania or in other catheter laboratories designated for $\mathrm{PH}$ patients. Acute vasodilator testing with nitric oxide using standard procedures and carefully monitored by an anesthesiologist was performed to identify the group of PAH patients who might benefit from Diltiazem. Ad-hoc left heart catheterization and/or coronary angiography were offered in selected cases.

Functional exercise capacity was objectively evaluated at admission using a non-encouraged 6MWD test performed according to American Thoracic Society Committee (ATS) and ERS/ATS recommendations and technical standards. ${ }^{28,29}$ The 6MWD and other features (pulse oximetry, heart rate, Borg dyspnea score before and immediately after the test) were recorded on standardized sheets.

As per the protocol, all available data were analyzed at three times: baseline and after an average of 7.5 and 13.5 months of uninterrupted specific therapy.

\section{Ethics Approval and Consent to Participate}

In compliance with the rules of the Helsinki Declaration and International Ethical Regulations, including all subsequent amendments, all study participants provided written informed consent. This study was conducted under the approval of the Research Ethics Committee of the "George Emil Palade" University of Medicine, Pharmacy, Science and Technology of Targu Mures, Romania (research approval number 1283/ 2021). 


\section{Statistical Analysis}

Data are shown as numerical values or percentages for categorical variables and were analyzed with the chisquare test (gender, WHO class [Table 1]). For continuous variables, the mean \pm standard deviation (SD) was calculated and analyzed with a parametric data test (ANOVA) for normally distributed data, and for skewed data, a nonparametric test Kruskal-Wallis (age, oxygen saturation [Table 1], cardiac index [Table 2]) was used. We considered a value of $\mathrm{p}<0.05$ to be statistically significant. The significance of the determinant and predictive effects of independent variables (etiology, age, oxygen saturation, red blood cell count, hemoglobin, hematocrit, iron deficiency status, NT-proBNP, and cardiac index) on dependent variable (6MWD) was assessed using univariate and multivariate linear regression models at baseline, 7.5 months and 13.5 months. The Kaplan-Meier method was used to estimate cumulative survival. ${ }^{30}$ All statistical analyses were carried out using GraphPad Prism version 9.00 (GraphPad Software, San Diego, CA, USA, www.graphpad.com) and IBM $^{\circledR} \quad$ SPSS $^{\circledR}$ Statistics for Windows version 25.0 (IBM Corp. Armonk, NY, USA).

Table I Patient's Baseline Characteristics and Subgroup Variables Analysis

\begin{tabular}{|c|c|c|c|c|c|c|}
\hline \multirow[t]{3}{*}{ Variable } & \multirow{3}{*}{$\begin{array}{l}\text { Patients } \\
(n=25)\end{array}$} & \multicolumn{4}{|c|}{ Clinical classification of $\mathrm{PH}$ groups } & \multirow{3}{*}{$\begin{array}{c}\text { p value } \\
\text { (ANOVA/*chi- } \\
\text { square test/ } \\
\text { **Kruskal- } \\
\text { Wallis test) }\end{array}$} \\
\hline & & \multicolumn{3}{|c|}{ Group I - PAH } & \multirow{2}{*}{$\begin{array}{c}\text { Group } 4 \text { - CTEPH } \\
\text { and other pulmonary } \\
\text { artery obstructions } \\
\text { Subgroup } \\
4.1 \text { - CTEPH } \\
(n=6)\end{array}$} & \\
\hline & & $\begin{array}{c}\text { Subgroup } \\
\begin{array}{c}I . I-i P A H \\
(n=5)\end{array}\end{array}$ & $\begin{array}{c}\text { Subgroup } \\
\text { I.4.I - PAH-CTD } \\
(n=2)\end{array}$ & $\begin{array}{c}\text { Subgroup } \\
\text { I.4.4 - PAH-CHD } \\
(n=I 2)\end{array}$ & & \\
\hline \multicolumn{7}{|l|}{ Clinical parameters } \\
\hline Age (years) & $46.40 \pm 18.64$ & $61.00 \pm 10.46$ & $51.00 \pm 1.41$ & $31.08 \pm 10.83$ & $63.33 \pm 13.89$ & $<0.000 I^{* *}$ \\
\hline Gender (female) & 15 & 4 & 1 & 9 & 1 & NS* \\
\hline BMI $\left(\mathrm{kg} / \mathrm{m}^{2}\right)$ & $25.17 \pm 5.42$ & $28.15 \pm 3.51$ & $20.60 \pm 0.57$ & $23.25 \pm 5.90$ & $28.05 \pm 4.26$ & NS \\
\hline WHO class (II/III/IV) & $4 / 17 / 4$ & $0 / 3 / 2$ & $1 / 1 / 0$ & $3 / 9 / 0$ & $0 / 4 / 2$ & $<0.0001 *$ \\
\hline 6MWD (meters) & $343 \pm 133$ & $230 \pm 96.4$ & $311 \pm 129$ & $424.2 \pm 80.5$ & $285 \pm 164$ & 0.0413 \\
\hline Oxygen saturation (\%, pulse oximetry) & $90.08 \pm 7.63$ & $94.20 \pm 4.81$ & $92.50 \pm 2.12$ & $86.50 \pm 9.20$ & $93.00 \pm 3.69$ & NS** \\
\hline $\mathrm{SBP}(\mathrm{mmHg})$ & $\mid 09.0 \pm 20.31$ & $115.0 \pm 25.98$ & $117.5 \pm 10.61$ & $101.7 \pm 20.71$ & $115.8 \pm 14.97$ & 0.4073 \\
\hline Heart rate (bpm) & $78.40 \pm 16.70$ & $74.60 \pm 7.30$ & $73.50 \pm 12.02$ & $80.92 \pm 18.42$ & $78.17 \pm 21.95$ & 0.8872 \\
\hline \multicolumn{7}{|c|}{ Laboratory parameters (normal values) } \\
\hline WBC $\left(4-9 \times 10^{3} / \mu L\right)$ & $7.81 \pm 1.65$ & $9.50 \pm 4.08$ & $6.33 \pm 0.79$ & $7.11 \pm 1.39$ & $8.28 \pm 1.86$ & 0.0137 \\
\hline $\operatorname{RBC}\left(4.2-5.5 \times 10^{6} / \mu \mathrm{L}\right)$ & $5.37 \pm 0.94$ & $5.23 \pm 0.96$ & $4.79 \pm 0.80$ & $5.62 \pm 1.03$ & $5.20 \pm 0.85$ & NS \\
\hline Hemoglobin (13.0-17.0 g/dL) & $14.86 \pm 2.79$ & $13.98 \pm 1.97$ & $12.85 \pm 1.20$ & $15.49 \pm 3.48$ & $15.02 \pm 2.01$ & NS \\
\hline Hematocrit (35.0-45.0\%) & $44.53 \pm 7.72$ & $41.82 \pm 5.16$ & $40.20 \pm 1.27$ & $46.63 \pm 9.80$ & $44.05 \pm 5.24$ & NS \\
\hline Serum iron $(9.0-30.0 \mu \mathrm{mol} / \mathrm{L})$ & $12.72 \pm 5.32$ & $11.7 \pm 4.39$ & $10.4 \pm 1.27$ & $12.80 \pm 5.66$ & $|4.18 \pm 6.6|$ & NS \\
\hline Serum ferritin $(\mu g / L)$ & $43.93 \pm 49.83$ & $34.36 \pm 25.93$ & $19.12 \pm 3.76$ & $32.06 \pm 35.52$ & $83.93 \pm 77.94$ & NS \\
\hline TSAT (\%) & $16.29 \pm 6.47$ & $14.38 \pm 4.72$ & $15.00 \pm 0.0$ & $15.97 \pm 7.01$ & $18.95 \pm 7.90$ & NS \\
\hline \multirow[t]{2}{*}{ NT-proBNP (min-max) (pg/mL) } & $1562 \pm 1645$ & $1836 \pm 2057$ & $|125 \pm| 4||$ & $937.4 \pm 1164$ & $2859 \pm 2077$ & NS \\
\hline & $(42.9-4980)$ & $(145-4780)$ & $(127.8-2123)$ & $(42.9-3800)$ & $(105-5760)$ & \\
\hline AST (5-45U/L) & $22.64 \pm 6.40$ & $23.60 \pm 8.82$ & $32.0 \pm 14.14$ & $21.17 \pm 3.74$ & $21.67 \pm 4.63$ & NS \\
\hline ALT (5-45U/L) & $17.32 \pm 7.57$ & $18.60 \pm 10.92$ & $14.50 \pm 3.54$ & $|5.92 \pm 5.8|$ & $20.0 \pm 9.03$ & NS \\
\hline $\mathrm{SCr}(0.5-1.0 \mathrm{mg} / \mathrm{dL})$ & $0.97 \pm 0.23$ & $1.07 \pm 0.21$ & $0.82 \pm 0.33$ & $0.87 \pm 0.14$ & $1.15 \pm 0.25$ & 0.0316 \\
\hline 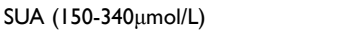 & $309.9 \pm 118.4$ & $329.80 \pm 49.66$ & $248.50 \pm 345.80$ & $301.8 \pm 97.27$ & $330 \pm 134.20$ & NS \\
\hline Fibrinogen $\left(1.70-3.20 \times 10^{2} \mathrm{mg} / \mathrm{dL}\right)$ & $4.06 \pm 0.59$ & $3.45 \pm 0.64$ & $3.38 \pm 0.92$ & $4.37 \pm 1.04$ & $4.06 \pm 0.59$ & NS \\
\hline
\end{tabular}

Notes: *Chi-square test; **Kruskal-Wallis test. Data are presented as mean \pm SD unless otherwise specified.

Abbreviations: ALT, alanine aminotransferase; AST, aspartate aminotransferase; BMI, body mass index; bpm, beats per minute; CTEPH, chronic thromboembolic pulmonary hypertension; iPAH, idiopathic pulmonary arterial hypertension; NS, not statistically significant; NT-proBNP, N-terminal pro-brain natriuretic peptide; PH, pulmonary hypertension; PAH, pulmonary arterial hypertension; PAH-CHD, pulmonary arterial hypertension associated with congenital heart disease; PAH-CTD, pulmonary arterial hypertension associated with connective tissue disease; RBC, red blood cells; SBP, systolic blood pressure; Scr, Serum creatinine; SUA, serum uric acid; TSAT, transferrin saturation; WBC, white blood cells; WHO, World Health Organization; 6MWD, six-minute walk distance. 


\section{Results}

Baseline characteristics and iron status for all 25 patients are summarized in Table 1. The male patients were on average older than the females $(p=0.03)$. The patients were mainly in WHO functional class III at baseline $(\mathrm{p}<0.0001)$.

With respect to $\mathrm{PH}$ etiology, there were no significant differences in the number of patients studied $(\mathrm{p}=0.05)$; however, the mean age was significantly different $(p<0.0001)$, CTEPH patients were older compared to the other groups (Table 1). Functional capacity assessed by 6MWD was significantly higher in the PAH-CHD subjects, with a mean value of $424.2 \pm 80.5 \mathrm{~m}$.

Invasive and non-invasive hemodynamic characteristics were assessed at the time of $\mathrm{PH}$ diagnosis by right heart catheterization and transthoracic echocardiography (Table 2). With respect to the mean PAPs, no significant statistical difference was observed between $\mathrm{PAH}$ and CTEPH patients $(\mathrm{p}=0.7889)$. The PH-CHD subgroup had the highest mean systolic pulmonary arterial pressure in the cohort, but this difference was not statistically significant $(p=0.0930)$. In the right atrium area analysis, two patients with a single atrium (belonging to subgroup 1.4.4) were excluded.

At baseline, $80.0 \%$ of iPAH patients ( 4 out of 5 patients) presented with pericardial effusion, which was significantly different from the other PH subgroups $(p=0.0029)$. One year mortality risk was assessed in the PAH group using an abridged REVEAL 2.0 risk calculator format (REVEAL Lite 2). ${ }^{15}$ Among 19 PAH patients, 94.73\% were considered at low-risk of mortality (Figure 1).

Treatment-naïve $\mathrm{PH}$ patients were started on initial monotherapy with $30 \%$ receiving endothelin receptor antagonists, 55\% phosphodiesterase-5 inhibitors, and $15 \%$ soluble guanylate cyclase. At the end of the study period, a double-sequential combination was prescribed to $60 \%$ of all patients. For PAH-CHD patients already receiving a specific treatment, the regimen was unchanged for entire study period.

Using the criteria described in Materials and Methods, iron deficiency was identified in 16 out of the 25 research subjects (Table 3 ).

Table 2 Hemodynamics, Cardiac Ultrasound Dataset, and Specific Therapy at Baseline in Studied Groups

\begin{tabular}{|c|c|c|c|c|c|c|}
\hline \multirow[t]{3}{*}{ Variable } & \multirow{3}{*}{$\begin{array}{l}\text { Patients } \\
(n=25)\end{array}$} & \multicolumn{4}{|c|}{ Clinical classification of $\mathrm{PH}$ groups } & \multirow{3}{*}{$p$ value } \\
\hline & & \multicolumn{3}{|c|}{ Group I - PAH } & \multirow{2}{*}{$\begin{array}{c}\text { Group } 4 \text { - CTEPH } \\
\text { and other } \\
\text { pulmonary artery } \\
\text { obstructions }\end{array}$} & \\
\hline & & $\begin{array}{c}\text { Subgroup } \\
\begin{array}{c}\text { I.I - iPAH } \\
(n=5)\end{array}\end{array}$ & $\begin{array}{c}\text { Subgroup } \\
\text { I.4.I - PAH- } \\
\text { CTD }(n=2)\end{array}$ & $\begin{array}{c}\text { Subgroup } \\
\text { I.4.4 - PAH-CHD } \\
(n=I 2)\end{array}$ & & \\
\hline \multicolumn{7}{|l|}{ Hemodynamic parameters (RHC) } \\
\hline mPAP $(\mathrm{mmHg})$ & $54.48 \pm 12.98$ & $60.2 \pm 10.08$ & $42 \pm 19.80$ & $58.08 \pm 9.33$ & $46.67 \pm 16.07$ & NS \\
\hline PVR (HRU) & $11.51 \pm 6.93$ & $11.5 \pm 9.72$ & $10.15 \pm 0.92$ & $12.79 \pm 7.68$ & $9.40 \pm 3.89$ & NS \\
\hline $\mathrm{Cl}\left(1 / \mathrm{min} / \mathrm{m}^{2}\right)$ & $2.70 \pm 1.00$ & $2.59 \pm 0.36$ & $2.40 \pm 0.14$ & $3.13 \pm 1.21$ & $2.09 \pm 0.73$ & NS \\
\hline \multicolumn{7}{|l|}{ Echocardiographic parameters } \\
\hline PAPs $(m m H g)$ & $81.26 \pm 21.73$ & $70.20 \pm 24.41$ & $54.50 \pm 31.82$ & $87.70 \pm|8.6|$ & $86.50 \pm 16.84$ & NS \\
\hline $\mathrm{RA}$ area $\left(\mathrm{cm}^{2}\right)$ & $24.59 \pm 10.08$ & $20.12 \pm 3.37$ & $22.00 \pm \mid 1.31$ & $29.92 \pm|9.5|$ & $33.32 \pm 13.43$ & NS \\
\hline IVC (mm) & $21.35 \pm 3.73$ & $22.2 \pm 2.78$ & $20.5 \pm 0.71$ & $20.98 \pm 4.45$ & $21.67 \pm 3.93$ & NS \\
\hline Pericardial effusion & 8 & 4 & 2 & 2 & - & 0.0029 \\
\hline Specific drug therapy & \multicolumn{6}{|c|}{ Patients (number) } \\
\hline ERA & 6 & - & 2 & 3 & 1 & - \\
\hline PDE5 & 11 & 5 & - & 4 & 2 & - \\
\hline Combined therapy (ERA and PDE5) & 5 & - & - & 5 & - & - \\
\hline sGC & 3 & - & - & - & 3 & - \\
\hline
\end{tabular}

Abbreviations: $\mathrm{Cl}$, cardiac index; $\mathrm{CTEPH}$, chronic thromboembolic pulmonary hypertension; ERA, endothelin receptor antagonists; $\mathrm{HRU}$, hybrid reference units (Wood units); iPAH, idiopathic pulmonary arterial hypertension; IVC, inferior vena cava; mPAP, mean pulmonary arterial pressure; n, number; NS, not statistically significant; PAH-CHD, pulmonary arterial hypertension associated with congenital heart disease; PAH-CTD, pulmonary arterial hypertension associated with connective tissue disease; PAH, pulmonary arterial hypertension; PAPs, pulmonary artery pressure (systolic); PDE5, phosphodiesterase type 5 inhibitors; PH, pulmonary hypertension; PVR, pulmonary vascular resistance; RA, right atrium; RHC, right heart catheterization; sGC, soluble guanylate cyclase. 


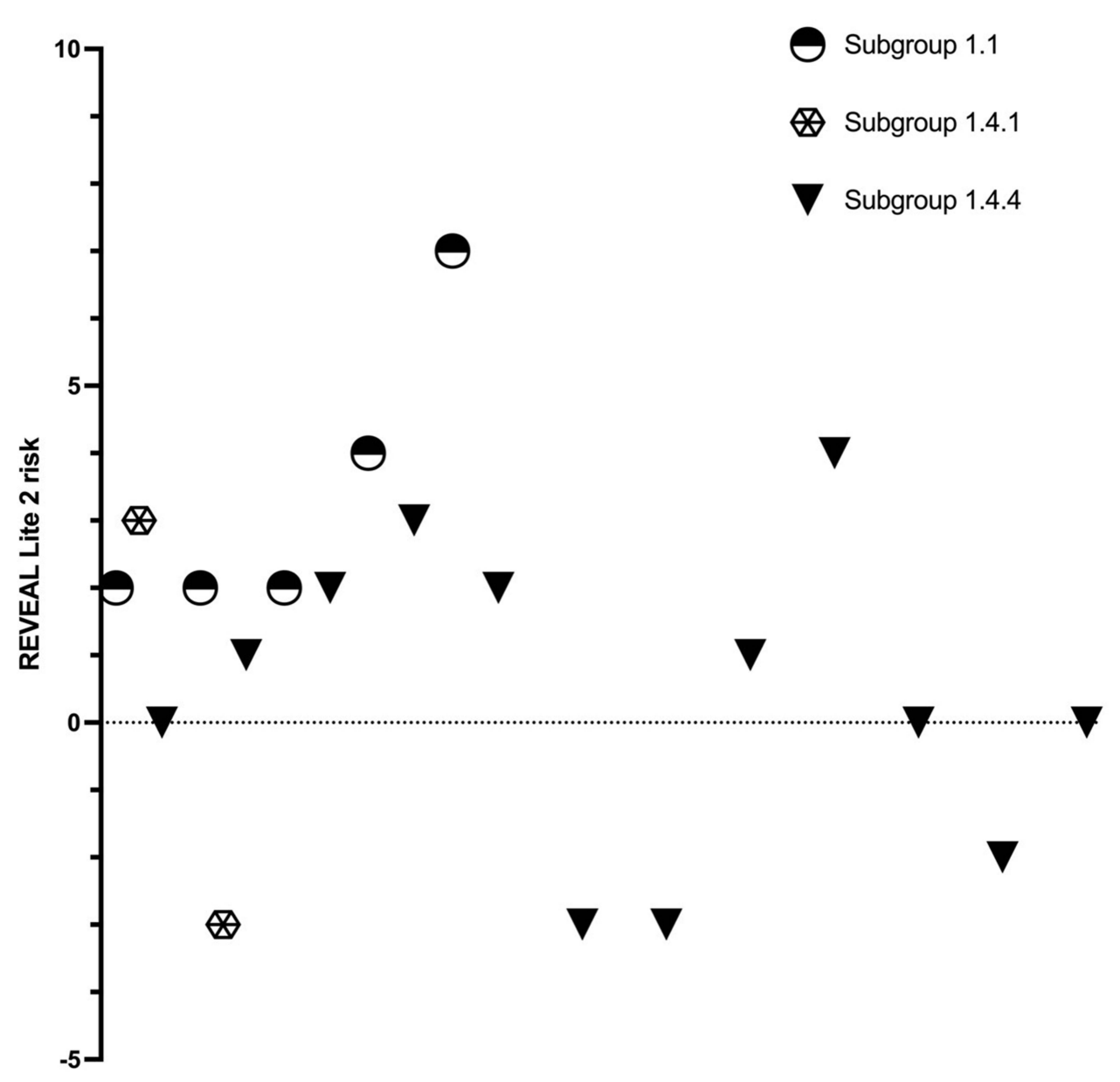

Figure I REVEAL Lite risk assessment of PAH patients (19 PAH patients ID and non-ID cases).

Abbreviations: ID, iron deficiency; PAH, pulmonary arterial hypertension; I.I, idiopathic pulmonary arterial hypertension; I.4.I, pulmonary arterial hypertensionassociated with connective tissue disease; 1.4.4, pulmonary arterial hypertension-associated with congenital heart disease.

The prevalence of iron deficiency was highest in the PAH-CTD subgroup (1.4.1). Iron-deficient patients were on average older than non-ID patients $(\mathrm{p}=0.8733)$. At baseline, more than half of ID patients $(56.25 \%)$ were in WHO functional class III. Functional capacity in IDpatients as assessed by 6MWD was $324.7 \pm 126.3$ meters, and although this was less than for the non-ID group (375.5 \pm 145.6 meters), the difference was not statistically significant $(\mathrm{p}=0.3702)$ (Figure 2).

Significantly lower levels of hemoglobin were identified in ID patients compared with non-ID patients $(13.69 \pm 1.602$ $\mathrm{g} / \mathrm{dL}$ vs $16.84 \pm 3.386 \mathrm{~g} / \mathrm{dL}$, respectively; $\mathrm{p}=0.0040$ ).

At baseline, univariate linear regression analysis did not show a statistically significant effect of ID on 6MWD $(p=0.428)$; ID accounts for only. $7 \%$ (coefficient of determination $\mathrm{R}^{2}=0.027$ ) of the total variation in the $6 \mathrm{MWD}$. In our univariate model, a decrease in the ID value by one unit corresponded to an increased walking distance of 3.805 meters.
In multivariate regression analysis at baseline, possible predictors of walking distance (etiology, age, peripheral oxygen saturation, red blood cell $[\mathrm{RBC}]$ count, hemoglobin and hematocrit values, iron deficiency status, NT-proBNP, and cardiac index) were not statistically significant $(\mathrm{p}=0.438)$. However, in the same analysis, the walking distance increased by $34.58 \mathrm{~m}$ in CTEPH patients, which correlated with reduced ID. In our model, the decrease in 6MWD was related to an increase in levels of NT-proBNP. Among the above-mentioned variables, four were predictive of 6MWD based on standardized coefficients (beta): cardiac index (0.319), hemoglobin (0.301), etiology (0.287), and iron deficiency $(-0.127)$.

In a similar multivariate regression model, we used data collected at a mean of 7.5 months to test for possible predictors of walking distance (excluding age, NTproBNP, and cardiac index). Correction of iron deficiency was associated with an increase in 6MWD of 10.644 


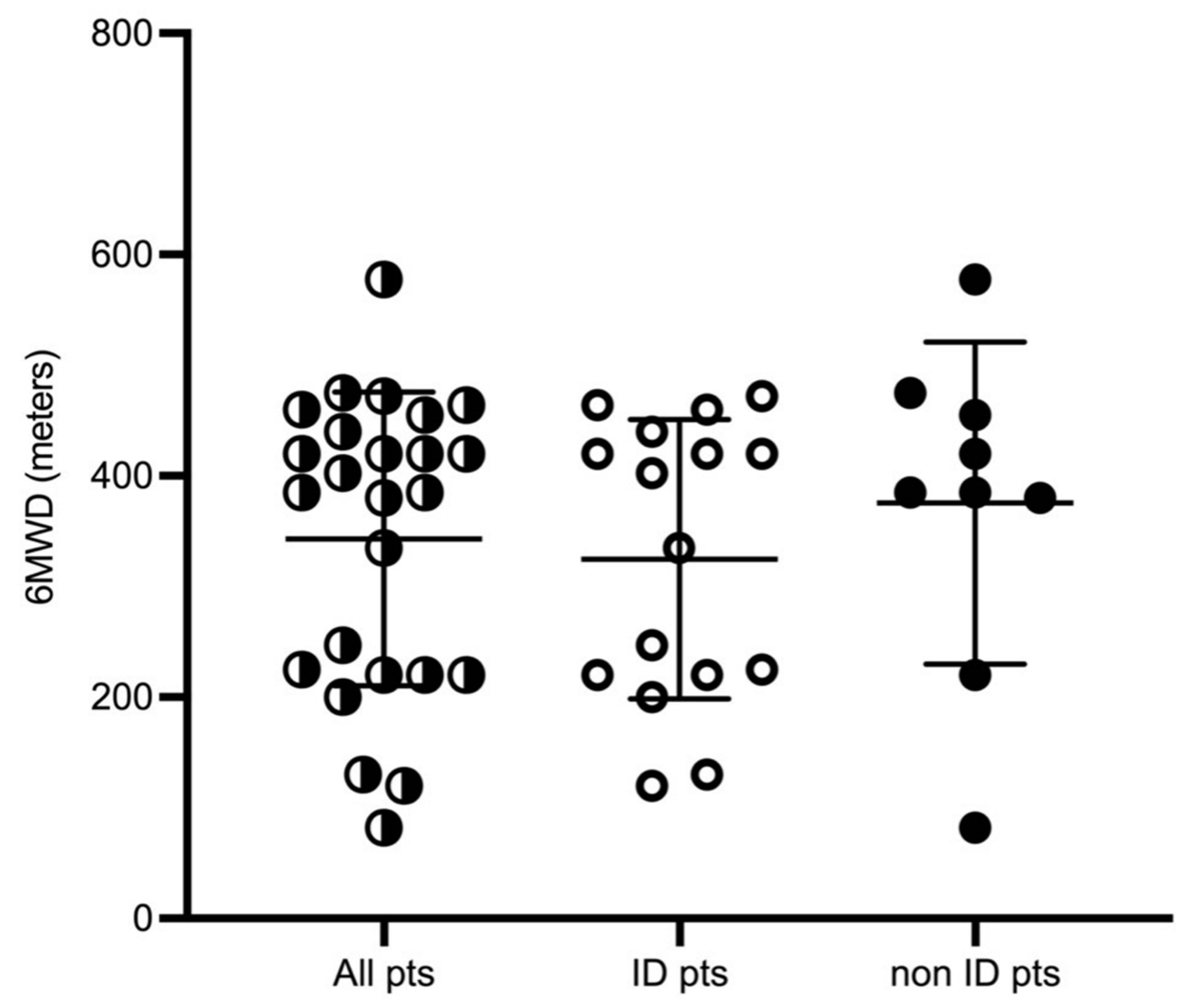

Figure 2 Six-minute walk distance in all studied case.

Abbreviations: 6MWD, six-minute walk distance; ID, iron deficiency; pts, patients.

meters in all patients. At this point, the most remarkable increase in 6MWD was related to PH etiology: compared with the baseline analysis, CTEPH patients experienced an increase of 58.66 meters compared with the PAH subgroups. Based on standardized coefficients (beta), the variables that were predictive of 6MWD were hematocrit (1.507), pulse oximetry (1.026), RBC count (0.926), and ID (-0.442).

At the end of the study (13.5 months), several predictive indices (ID, age, etiology, pulse oximetry, RBC value, hemoglobin, and NT-proBNP), and the dependent variable 6MWD were analyzed in a multivariate regression model. This analysis did not show statistical significance $(\mathrm{p}=0.361)$, and ID did not exert the same influence on 6MWD as in the other models ( $p=0.972$ ), independent of ID correction. Similarly, the mean value for the NT-proBNP cohort was not significantly different $(\mathrm{p}=0.4678)$, despite the individual difference $(\mathrm{p}=0.0002)$, at baseline and the end of the study.

Survival was assessed in February 2021, five years after the first patient was enrolled. The overall survival rate was $76.0 \%$ at 60 months follow-up. Three patients could not be followed up throughout the entire study. Four
PAH patients that were low-risk (REVEAL Lite score) died; we recorded two deaths unrelated to cardiac conditions (one case from a brain tumor and one case from a domestic accident); one patient presenting intermediate risk of death ( 7 points) died 5 months after enrollment. One death in the CTEPH group was recorded in a 79 yearold male high-risk patient (ESC/ERS risk assessment). Survival rate $(\mathrm{p}=0.5804)$ is presented in Figure 3.

\section{Discussion}

In Romania, pulmonary hypertension assessment and management currently occurs at seven designated referral centers. The Romanian National Program for Treatment of Pulmonary Hypertension started in 2008 and remains under development. The incidence of PH cases in our center (5 cases in 2015, rising to a total of 48 targeted therapy cases in 2021) is comparable to previously published data from registries covering several European countries and the USA. ${ }^{31-37}$

As of 2018, 2967 PAH patients in the largest database (REVEAL Registry) had a mean age of $53 \pm 14$ years, higher than in our study $(46.40 \pm 18.64$ years), 
Table 3 Iron Deficiency-Patient Characteristics and Subgroup Variables at Baseline

\begin{tabular}{|c|c|c|c|c|c|c|}
\hline & \multirow{3}{*}{ Patients } & \multicolumn{5}{|c|}{ Clinical classification of PH groups } \\
\hline & & \multicolumn{3}{|c|}{ Group I - PAH } & \multirow{2}{*}{$\begin{array}{c}\text { Group } 4 \text { - CTEPH } \\
\text { and other } \\
\text { pulmonary artery } \\
\text { obstructions }\end{array}$} & \multirow[t]{2}{*}{ p value } \\
\hline & & $\begin{array}{c}\text { Subgroup } \\
I . I-i P A H \\
(n=5)\end{array}$ & $\begin{array}{c}\text { Subgroup } \\
\text { I.4.I - PAH-CTD } \\
(n=2)\end{array}$ & $\begin{array}{c}\text { Subgroup } \\
\begin{array}{c}\text { I.4.4 - PAH-CHD } \\
(n=12)\end{array}\end{array}$ & & \\
\hline ID patients /all studied patients & $16 / 25$ & $3 / 5$ & $2 / 2$ & $8 / 12$ & $3 / 6$ & NS \\
\hline ID percentage & $64.0 \%$ & $60.0 \%$ & $100 \%$ & $66.66 \%$ & $50.0 \%$ & NS \\
\hline Variables & ID patients & \multicolumn{5}{|c|}{ Iron deficiency in subgroups } \\
\hline WHO functional class (I/II/III/IV) & $0 / 4 / 9 / 3$ & $0 / 0 / 1 / 2$ & $0 / 1 / 1 / 0$ & $0 / 3 / 5 / 0$ & $0 / 0 / 2 / 1$ & NS \\
\hline 6MWD (meters) & $324.7 \pm 126.3$ & $181.7 \pm 54.85$ & $311.3 \pm 129.0$ & $407.3 \pm 77.91$ & $256.7 \pm 148.4$ & 0.0206 \\
\hline Oxygen saturation (\%) & $89.31 \pm 7.43$ & $92.00 \pm 5.29$ & $92.50 \pm 2.12$ & $86.0 \pm 8.90$ & $93.33 \pm 4.16$ & NS \\
\hline Serum iron $(\mu g / L)$ & $9.61 \pm 2.85$ & $9.40 \pm 1.74$ & $10.40 \pm 1.27$ & $9.86 \pm 3.67$ & $8.63 \pm 2.74$ & NS \\
\hline Serum ferritin $(\mathrm{ng} / \mathrm{mL})$ & $16.16 \pm 3.67$ & $17.46 \pm 2.98$ & $19.12 \pm 3.76$ & $14.95 \pm 4.14$ & $16.12 \pm 2.79$ & NS \\
\hline TSAT (\%) & $12.26 \pm 2.66$ & $11.30 \pm 2.25$ & $15.0 \pm 0.0$ & $11.70 \pm 2.34$ & $12.90 \pm 4.25$ & NS \\
\hline Hemoglobin $(g / d L)$ & $13.68 \pm 1.60$ & $13.17 \pm 2.93$ & $12.85 \pm 1.20$ & $14.11 \pm 1.52$ & $13.57 \pm 0.23$ & NS \\
\hline NT-proBNP (pg/mL) & $1684 \pm 1813$ & $2895 \pm 2061$ & $|125 \pm| 4||$ & $824.10 \pm 123$ & $3139 \pm 2340$ & NS \\
\hline
\end{tabular}

Abbreviations: PH, pulmonary hypertension; PAH, pulmonary arterial hypertension; ID, iron deficiency, iPAH, idiopathic pulmonary arterial hypertension; PAH-CTD, pulmonary arterial hypertension associated with connective tissue disease; $\mathrm{PAH}-\mathrm{CHD}$, pulmonary arterial hypertension associated with congenital heart disease; CTEPH, chronic thromboembolic pulmonary hypertension; ID, iron deficiency; NS, not statistically significant; NT-proBNP, N-terminal pro-brain natriuretic peptide; TSAT, transferrin saturation; WHO, World Health Organization; 6MWD, six-minute walk distance; NS, not statistically significant.

and females outnumbered males, ${ }^{38}$ consistent with our study in which the proportions were $79.5 \%$ vs $60.0 \%$, respectively.
In the present study, an unexpectedly high proportion $(84.0 \%)$ of $\mathrm{PH}$ patients were in WHO functional classes III or IV at the time of their diagnosis, with $68.0 \%$ in class III.

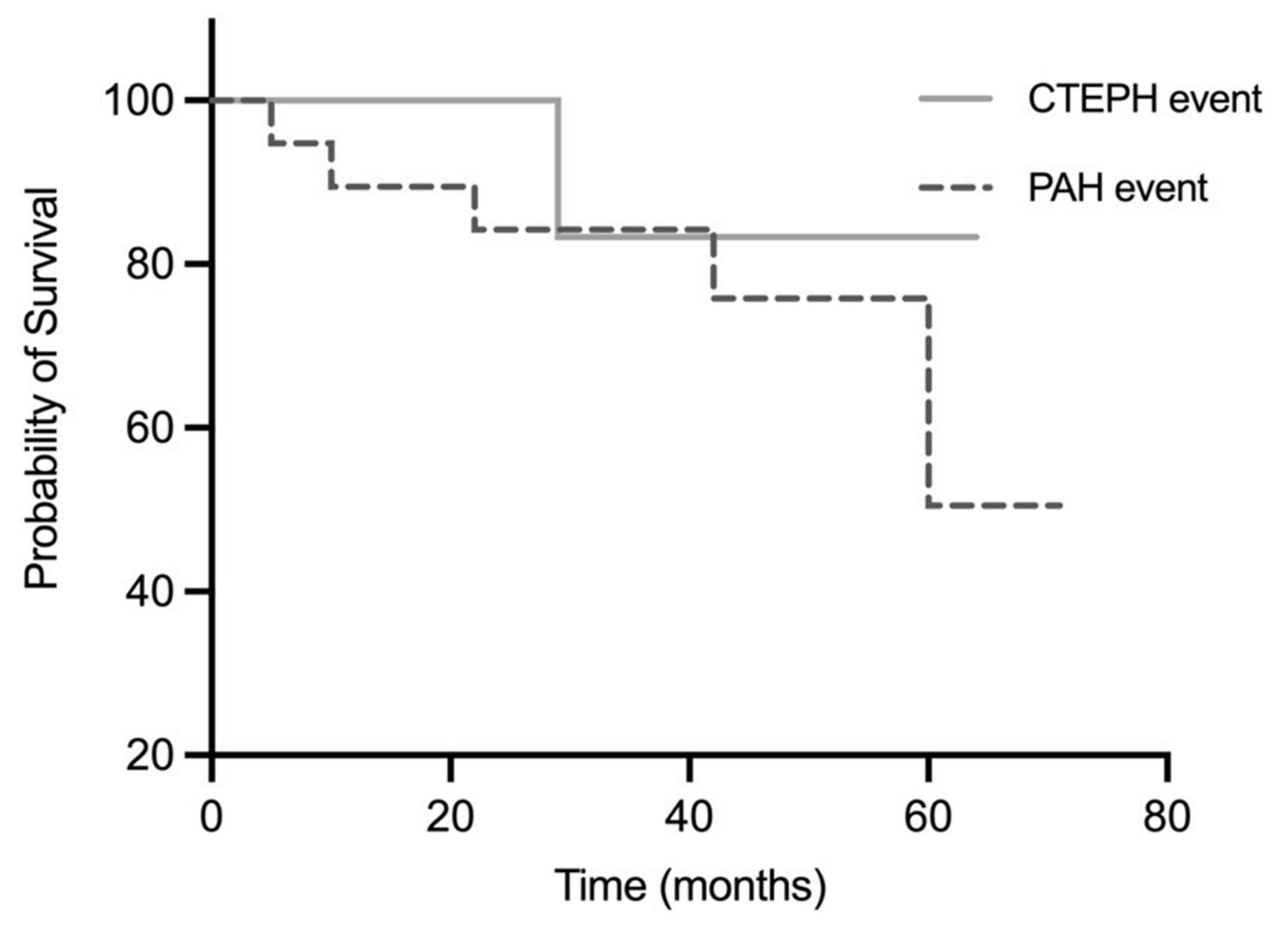

Figure 3 Survival rate at 60 months in PAH and CTEPH cases.

Abbreviations: CTEPH, chronic thromboembolic pulmonary hypertension; $\mathrm{PAH}$, pulmonary arterial hypertension. 
These results exceed those in the French Pulmonary Hypertension Registry published by Humbert et al. ${ }^{39}$ Our results might be partially explained by a relative lack of awareness of this disease by practitioners, and patients being slow to present themselves to the medical services.

A notable feature of the current study was the marked prevalence of PAH-CHD (12/25 patients, $48.0 \%$ ), which is greater than that in a Chinese study published in 2018, where a percentage of $31.07 \%$ was reported in the 1.4.4-PAH subgroup. ${ }^{40}$ Data in a Portuguese registry (2017) exhibited a much lower prevalence $(20.9 \%)$ for PAH etiology associated with congenital heart disease. ${ }^{41}$

An additional observation of the current research refers to the lower age of the study group ( $46.40 \pm 18.64$ years) compared with data published in some European and US studies that reported greater age at the time of diagnosis. ${ }^{42}$ Chazova et al reported in 2020 that Russian newly diagnosed PAH patients were significantly younger $(45.2 \pm 14.9$ years) than CTEPH patients (52.6 \pm 15.3 years). ${ }^{5}$ The large number of PAH-CHD patients partially explains such age differences in our study, where $48.0 \%$ of all subjects were aged 18 years old, having been referred from the pediatric $\mathrm{PH}$ centre based at the same university. Another factor is the limited availability of corrective surgery in congenital heart disease patients in Romania. In our study, PAH-CTD patients were underrepresented, which can be explained by the low number of referrals from rheumatology units, similar to the data published by Radchenko et al in $2019 .{ }^{43}$

Even though pulmonary endarterectomy is the first-line treatment for CTEPH patients, none of our patients underwent this procedure due to a lack of PEA centers in our country. ${ }^{1,44}$ Balloon pulmonary angioplasty was performed in our center on two inoperable patients, being the first in Romania (2018). A multidisciplinary team (PH-dedicated clinical cardiologist, interventional cardiologist, anesthesiologist, and cardiac surgeon) assessed the inoperability under the supervision of an experienced external $\mathrm{PH}$ team from Poland. The two patients benefitted from BPA while their therapeutical regimen (Riociguat $2.5 \mathrm{mg}$ t.i.d.) was continued.

Assessment of iron deficiency is primarily based on the measurement of serum ferritin and TSAT values, with some limitations. ${ }^{45,46}$ These parameters can be affected by a variety of factors such as iron stores in the body, chronic diseases with significant inflammatory components, or infections. ${ }^{47}$ Sonnweber et al pointed out that the levels of serum transferrin receptor (sTFR), the sTFR/ $\log$ ferritin index (sTFRF) and serum hepcidin can be measured for a more precise description of ID. ${ }^{48}$

Regarding iron deficiency in different subgroups of pulmonary hypertension patients, the results of our study partially overlap with those of Yu et al (2018), who acknowledged the prevalence of iron deficiency in PAHCTD patients and its low incidence in CTEPH cases. ${ }^{40}$ Other studies in which only $4.9 \%$ of CTEPH patients were iron deficient contrast with our findings that half of CTEPH patients were iron deficient. ${ }^{49}$ This high percentage can be explained by the characteristics of our study.

The difference in the mean age in the ID group compared with that of the non-ID patients contrasts with data published by Soon et al for which there was a lower mean age for patients with PAH who were iron-deficient. ${ }^{49}$ An explanation for this difference may lie in the small sample and heterogeneous PH etiologies in our study, as well as the enrollment of premenopausal women in the Soon et al study vs postmenopausal women in ours. By comparing the mean iron deficiency values between the four $\mathrm{PH}$ subgroups, we found that subgroup 1.4.4 had the highest ID as indicated by the lowest ferritin values $(14.95 \pm 4.14$ $\mathrm{pmol} / \mathrm{L}$ ). This finding contrasts with the data published in 2018 by $\mathrm{Yu}$ et al who reported the highest prevalence of iron deficiency in PAH-CTD patients. ${ }^{40}$

PAH patients are frequently iron deficient. ${ }^{50}$ Oral iron therapy cannot restore normal iron status due to reduced gastrointestinal absorption and a high rate of adverse events. ${ }^{49}$ Under these circumstances, parenteral iron supplementation is needed as shown by Viethen et al. ${ }^{19,49}$

$\mathrm{Xi}$ et al showed that CTEPH is not associated with iron metabolism. ${ }^{51}$ Conversely, CTEPH patients are often in the advanced stages of heart failure, for which iron replacement therapy is beneficial. ${ }^{44,45}$ Based on this information, we took a cautious approach to correcting iron deficiency, taking into consideration possible endothelial dysfunction, intensification of atherosclerosis, and progression of kidney injury. ${ }^{18}$

Van Empel et al stated that NYHA functional class III and higher rates of heart failure were associated with iron deficiency, results similar to our study in which $75 \%$ of ID patients were classified as WHO functional classes III and $\mathrm{IV}^{52}$ Van Empel et al also reported that iron-deficient patients exhibited 6MWD comparable to that of non-ID patients, ${ }^{52}$ similar to our results.

Viethen et al showed that in PAH patients, restoring normal iron status was associated with a clinically meaningful improvement in exercise capacity. This benefit was 
in addition to that provided by specific PAH therapy. ${ }^{19}$ The results of our study showed that improvement of iron stores was correlated with an increase in walking distance; moreover, after ID correction, after 13.5 months of evaluation, 6MWD increased significantly compared with that at the time of patient recruitment. The effect of iron deficiency on functional capacity, as judged by $6 \mathrm{MWD}$, was observed in all iron-deficient PH groups. However, the greatest benefit of ID correction was observed in the CTEPH subgroup. Medium and long-term survival of iPAH patients is also related to iron status and anemia. ${ }^{53}$

\section{Study Limitations}

We acknowledge that this research has several significant limitations related to its design. It is a retrospective study using available paper-based and electronic data from a single center. It represents a population-based study and is comprised of a small sample from PAH and CTEPH subgroups across Romania. However, the actual incidence of the two PH subgroups in the general population is unknown. A second important limitation arises from the large proportion of PAH-CHD patients (12 out of 25 patients). Data on transferrin saturation, serum hepcidin and soluble transferrin receptor values were not routinely available. Furthermore, hemodynamic follow-up was not available at well-defined intervals due to the inconstant availability of a cardiac catheterization facility.

Despite the inherent limitations (small study, single center, uncontrolled, limited data availability), the present analysis highlights the importance of continued collaborative research between experienced large $\mathrm{PH}$-expert centers and newly organized small facilities.

\section{Conclusion}

This study provides the first published data from Romania on the presence of iron deficiency in different subgroups of precapillary PH patients. Patients with PH-CTD (subgroup 1.4.1) have an increased prevalence of iron deficiency compared with other etiologies (subgroups 1.1, 1.4.4, and 4.1).

The results of this study demonstrate that iron deficiency has a negative impact on the functional status (assessed by 6MWD), in specific groups and subgroups of patients with precapillary pulmonary hypertension, albeit not independently nor significant to other known predictors such as age, gender, oxygen saturation, and hemoglobin value.

These data can be integrated with global research and are consistent with phenotypes of patients diagnosed with pulmonary hypertension of different etiologies.

\section{Data Sharing Statement}

All data accompanying the present study are available from the corresponding author upon reasonable request.

\section{Acknowledgments}

We thank all patients and collaborators who were involved in this study. Special thanks to Professor Irene Marthe Lang from Department of Internal Medicine II, Division of Cardiology, Medical University of Vienna, Professor Adam Torbicki, and Professor Marcin Kurzyna from Department of Pulmonary Circulation, Thromboembolic Diseases and Cardiology, Centre of Postgraduate Medical Education, European Health Centre, Otwock, Poland for their invaluable encouragement, education and training of the $\mathrm{PH}$ team from Targu Mures, Romania, and their support of the first BPA's in Romania.

\section{Author Contributions}

All authors made substantial contributions to the conception and design of the study, and participated in acquisition or analysis and interpretation of data, took part in drafting or critically revising the article to optimize it for important intellectual content, agreed to submit to the current journal, gave final approval for this version to be published, and agree to be accountable for all aspects of the work.

\section{Funding}

This work was partially supported by the "George Emil Palade" University of Medicine, Pharmacy, Science and Technology of Targu Mures, Romania, Research Grant number 615/13/17.01.2019.

\section{Disclosure}

The authors declare that they have no conflicts of interest related to this study.

\section{References}

1. Galiè N, Humbert M, Vachiery JL, et al.; ESC Scientific Document Group. 2015 ESC/ERS Guidelines for the diagnosis and treatment of pulmonary hypertension: the joint task force for the diagnosis and treatment of pulmonary hypertension of the European Society of Cardiology (ESC) and the European Respiratory Society (ERS): endorsed by: association for European Paediatric and Congenital Cardiology (AEPC), International Society for Heart and Lung Transplantation (ISHLT). Eur Heart J. 2016;37(1):67-119. doi:10.1093/eurheartj/ehv317.

2. Galiè N, Channick RN, Frantz RP, et al. Risk stratification and medical therapy of pulmonary arterial hypertension. Eur Respir J. 2019;53 (1):1801889. doi:10.1183/13993003.01889-2018

3. Simonneau G, Montani D, Celermajer DS, et al. Haemodynamic definitions and updated clinical classification of pulmonary hypertension. Eur Respir J. 2019;53(1):1801913. doi:10.1183/ 13993003.01913-2018 
4. Jansa P, Jarkovsky J, Al-Hiti H, et al. A Epidemiology and long-term survival of pulmonary arterial hypertension in the Czech Republic: a retrospective analysis of a nationwide registry. BMC Pulm Med. 2014;14:(1):45. doi:10.1186/1471-2466-14-45

5. Chazova IY, Martynyuk TV, Valieva ZS, et al. Clinical and Instrumental Characteristics of newly diagnosed patients with various forms of pulmonary hypertension according to the Russian National Registry. Biomed Res Int. 2020;2020:6836973. doi:10.1155/2020/ 6836973

6. Kigitovica D, Sablinskis M, Sablinskis K, et al. Pulmonary arterial hypertension and chronic thromboembolic pulmonary hypertension incidence in Latvia in 2018. Eur J Intern Med. 2019;65:e9-e10. doi:10.1016/j.ejim.2019.04.022

7. Benza RL, Miller DP, Foreman AJ, et al. Prognostic implications of serial risk score assessments in patients with pulmonary arterial hypertension: a Registry to Evaluate Early and Long-Term Pulmonary Arterial Hypertension Disease Management (REVEAL) analysis. J Heart Lung Transplant. 2015;34(3):356-361. doi:10.1016/ j.healun.2014.09.016

8. Benza RL, Gomberg-Maitland M, Elliott CG, et al. Predicting survival in patients with pulmonary arterial hypertension: the REVEAL risk score calculator.0 and comparison With ESC/ERS-based risk assessment strategies. Chest. 2019;156(2):323-337. doi:10.1016/j. chest.2019.02.004

9. Kanwar M, Raina A, Lohmueller L, et al. The use of risk assessment tools and prognostic scores in managing patients with pulmonary arterial hypertension. Curr Hypertens Rep. 2019;21(6):45. doi:10.1007/s11906-019-0950-y

10. Sitbon O, Nikkho S, Benza R, et al. Novel composite clinical endpoints and risk scores used in clinical trials in pulmonary arterial hypertension. Pulm Circ. 2020;10(4):2045894020962960. doi:10.1177/2045894020962960

11. Sonnweber T, Schneider EM, Nairz M, et al. Risk assessment in precapillary pulmonary hypertension: a comparative analysis. Respir Res. 2021;22(1):28. doi:10.1186/s12931-021-01624-z

12. Boucly A, Weatherald J, Savale L, et al. Risk assessment, prognosis and guideline implementation in pulmonary arterial hypertension. Eur Respir J. 2017;50(2):1700889. doi:10.1183/13993003.008892017

13. Xiong W, Zhao Y, Xu M, et al. A modified risk score in one-year survival rate assessment of group 1 pulmonary arterial hypertension. BMC Pulm Med. 2018;18(1):161. doi:10.1186/s12890-018-0712-7

14. Deng X, Jin B, Li S, et al. Guideline implementation and early risk assessment in pulmonary arterial hypertension associated with congenital heart disease: a retrospective cohort study. Clin Respir J. 2019;13(11):693-699. doi:10.1111/crj.13076

15. Benza RL, Kanwar MK, Raina A, et al. Development and validation of an abridged version of the REVEAL.0 risk score calculator, REVEAL Lite, for Use in patients with pulmonary arterial hypertension. Chest. 2021;159(1):337-346. doi:10.1016/j. chest.2020.08.2069

16. Ntiloudi D, Zanos S, Gatzoulis MA, et al. How to evaluate patients with congenital heart disease-related pulmonary arterial hypertension. Expert Rev Cardiovasc Ther. 2019;17(1):11-18. doi:10.1080/ 14779072.2019.1550716

17. Rhodes CJ, Howard LS, Busbridge M, et al. Iron deficiency and raised hepcidin in idiopathic pulmonary arterial hypertension: clinical prevalence, outcomes, and mechanistic insights. $\mathrm{J} \mathrm{Am} \mathrm{Coll} \mathrm{Cardiol}$. 2011;58(3):300-309. doi:10.1016/j.jacc.2011.02.057

18. Ghio S, Fortuni F, Capettini AC, et al. Iron deficiency in pulmonary arterial hypertension: prevalence and potential usefulness of oral supplementation. Acta Cardiol. 2020;1-6.

19. Viethen T, Gerhardt F, Dumitrescu D, et al. Ferric carboxymaltose improves exercise capacity and quality of life in patients with pulmonary arterial hypertension and iron deficiency: a pilot study. Int J Cardiol. 2014;175(2):233-239. doi:10.1016/j.ijcard.2014.04.233
20. Broberg CS, Bax BE, Okonko DO, et al. Blood viscosity and its relationship to iron deficiency, symptoms, and exercise capacity in adults with cyanotic congenital heart disease. $J$ Am Coll Cardiol. 2006;48(2):356-365. doi:10.1016/j.jacc.2006.03.040

21. Thomas DW, Hinchliffe RF, Briggs C, Macdougall IC, Littlewood T, Cavill I. British committee for standards in haematology. Guideline for the laboratory diagnosis of functional iron deficiency. $\mathrm{Br}$ J Haematol. 2013;161(5):639-648. doi:10.1111/bjh.12311

22. Yamanishi H, Iyama S, Yamaguchi Y, Kanakura Y, Iwatani Y. Total iron-binding capacity calculated from serum transferrin concentration or serum iron concentration and unsaturated iron-binding capacity. Clin Chem. 2003;49(1):175-178. doi:10.1373/49.1.175

23. Sonnweber T, Nairz M, Theurl I, et al. The crucial impact of iron deficiency definition for the course of precapillary pulmonary hypertension. PLoS One. 2018;13(8):e0203396. doi:10.1371/journal. pone. 0203396

24. Koch TA, Myers J, Goodnough LT. Intravenous iron therapy in patients with iron deficiency anemia: dosing considerations. Anemia. 2015;2015:763576. doi:10.1155/2015/763576

25. Steeds RP, Garbi M, Cardim N, et al.; 014-2016 EACVI Scientific Documents Committee;014-2016 EACVI Scientific Documents Committee. EACVI appropriateness criteria for the use of transthoracic echocardiography in adults: a report of literature and current practice review. Eur Heart J Cardiovasc Imaging. 2017;18 (11):1191-1204. doi:10.1093/ehjci/jew333.

26. Porter TR, Shillcutt SK, Adams MS, et al. Guidelines for the use of echocardiography as a monitor for therapeutic intervention in adults: a report from the American Society of Echocardiography. $J \quad$ Am Soc Echocardiogr. 2015;28(1):40-56. doi:10.1016/j. echo.2014.

09.009

27. Rosenkranz S, Preston IR. Right heart catheterisation: best practice and pitfalls in pulmonary hypertension. Eur Respir Rev. 2015;24 (138):642-652. doi:10.1183/16000617.0062-2015

28. ATS Committee on Proficiency Standards for Clinical Pulmonary Function Laboratories. ATS statement: guidelines for the six-minute walk test. Am J Respir Crit Care Med. 2002;166(1):111-117. doi:10.1164/ajrccm.166.1.at1102

29. Holland AE, Spruit MA, Troosters T, et al. An official European Respiratory Society/American Thoracic Society technical standard: field walking tests in chronic respiratory disease. Eur Respir J. 2014;44(6):1428-1446. doi:10.1183/09031936.00150314

30. Kaplan EL, Meier P. Nonparametric estimation from incomplete observations. J Am Stat Assoc. 1958;53(282):457-481. doi:10.1080/ 01621459.1958 .10501452

31. Olsson KM, Delcroix M, Ghofrani HA, et al. Anticoagulation and survival in pulmonary arterial hypertension: results from the Comparative, Prospective Registry of Newly Initiated Therapies for Pulmonary Hypertension (COMPERA). Circulation. 2014;129 (1):57-65. doi:10.1161/CIRCULATIONAHA.113.004526

32. Pittrow D, Ghofrani HA, Opitz CF, Huscher D, Hoeper MM. Internationales, prospektives Register zur Erfassung der Initial- und Dauertherapie von Patienten mit pulmonaler Hypertonie (CompERA-XL) [International, prospective register for the documentation of first-line and maintenance therapy in patients with pulmonary hypertension (CompERA-XL)]. Dtsch Med Wochenschr. 2009;134 Suppl 5(Suppl 5):S173-175. doi:10.1055/s-0029-1225318

33. Peacock AJ, Murphy NF, McMurray JJ, Caballero L, Stewart S. An epidemiological study of pulmonary arterial hypertension. Eur Respir J. 2007;30(1):104-109. doi:10.1183/09031936.00092306

34. Baptista R, Meireles J, Agapito A, et al. Pulmonary hypertension in Portugal: first data from a nationwide registry. Biomed Res Int. 2013;2013:489574. doi:10.1155/2013/489574

35. Rich S, Dantzker DR, Ayres SM, et al. Primary pulmonary hypertension. A national prospective study. Ann Intern Med. 1987;107 (2):216-223. doi:10.7326/0003-4819-107-2-216 
36. Rådegran G, Kjellström B, Ekmehag B, et al.; on behalf of SveFPH and SPAHR. Characteristics and survival of adult Swedish PAH and CTEPH patients000-2014. Scand Cardiovasc J. 2016;50(4):243-250. doi:10.1080/14017431.2016.1185532.

37. Escribano-Subias P, Blanco I, López-Meseguer M, et al. REHAP investigators. Survival in pulmonary hypertension in Spain: insights from the Spanish registry. Eur Respir J. 2012;40(3):596-603. doi:10.1183/09031936.00101211

38. Badesch DB, Raskob GE, Elliott CG, et al. Pulmonary arterial hypertension: baseline characteristics from the REVEAL Registry. Chest. 2010;137(2):376-387. doi:10.1378/chest.09-1140

39. Humbert M, Sitbon O, Chaouat A, et al. Pulmonary arterial hypertension in France: results from a national registry. Am J Respir Crit Care Med. 2006;173(9):1023-1030. doi:10.1164/ rccm.200510-16680C

40. Yu X, Luo Q, Liu Z, et al. Prevalence of iron deficiency in different subtypes of pulmonary hypertension. Heart Lung. 2018;47 (4):308-313. doi:10.1016/j.hrtlng.2018.05.002

41. Marques-Alves P, Baptista R, Marinho da Silva A, Pêgo M, Castro G. Real-world, long-term survival of incident patients with pulmonary arterial hypertension. Rev Port Pneumol. 2017;23(3):124-131. doi:10.1016/j.rppnen.2017.01.006

42. Hoeper MM, Huscher D, Ghofrani HA, et al. Elderly patients diagnosed with idiopathic pulmonary arterial hypertension: results from the COMPERA registry. Int $J$ Cardiol. 2013;168(2):871-880. doi:10.1016/j.ijcard.2012.10.026

43. Radchenko GD, Zhyvylo IO, Sirenko YM. Analysis of pulmonary hypertension patient survival after treatment in referral center (data of first Ukrainian register). Pulm Circ. 2019;9(2):2045894019845604. doi:10.1177/2045894019845604

44. Kim NH, Delcroix M, Jais X, et al. Chronic thromboembolic pulmonary hypertension. Eur Respir J. 2019;53(1):1801915. doi:10.1183/13993003.01915-2018
45. Ponikowski P, van Veldhuisen DJ, Comin-Colet J, et al. CONFIRMHF Investigators. Beneficial effects of long-term intravenous iron therapy with ferric carboxymaltose in patients with symptomatic heart failure and iron deficiency. Eur Heart J. 2015;36 (11):657-668. doi:10.1093/eurheartj/ehu385

46. Anker SD, Comin Colet J, Filippatos G, et al. FAIR-HF Trial Investigators. Ferric carboxymaltose in patients with heart failure and iron deficiency. $N$ Engl J Med. 2009;361(25):2436-2448. doi:10.1056/NEJMoa0908355

47. Ginzburg YZ. New diagnostic tools for delineating iron status. Hematology Am Soc Hematol Educ Program. 2019;2019 (1):327-333. doi:10.1182/hematology.2019000035

48. Sonnweber T, Pizzini A, Tancevski I, et al. Anaemia, iron homeostasis and pulmonary hypertension: a review. Intern Emerg Med. 2020;15(4):573-585. doi:10.1007/s11739-020-02288-1

49. Soon E, Treacy CM, Toshner MR, et al. Unexplained iron deficiency in idiopathic and heritable pulmonary arterial hypertension. Thorax. 2011;66(4):326-332. doi:10.1136/thx.2010.147272

50. Ruiter G, Lankhorst S, Boonstra A, et al. Iron deficiency is common in idiopathic pulmonary arterial hypertension. Eur Respir J. 2011;37 (6):1386-1391. doi:10.1183/09031936.00100510

51. Xi Q, Liu Z, Liu W, Zhao Z, Luo Q, Huang Z. Chronic thromboembolic pulmonary hypertension is not associated with iron overload. Cardiovasc Pathol. 2015;24(2):76-79. doi:10.1016/j. carpath.2014.09.006

52. Van Empel VP, Lee J, Williams TJ, Kaye DM. Iron deficiency in patients with idiopathic pulmonary arterial hypertension. Heart Lung Circ. 2014;23(3):287-292. doi:10.1016/j.hlc.2013.08.007

53. Rhodes CJ, Wharton J, Howard L, Gibbs JS, Vonk-Noordegraaf A, Wilkins MR. Iron deficiency in pulmonary arterial hypertension: a potential therapeutic target. Eur Respir J. 2011;38(6):1453-1460. doi:10.1183/09031936.00037711
International Journal of General Medicine

\section{Publish your work in this journal}

The International Journal of General Medicine is an international, peer-reviewed open-access journal that focuses on general and internal medicine, pathogenesis, epidemiology, diagnosis, monitoring and treatment protocols. The journal is characterized by the rapid reporting of reviews, original research and clinical studies

\section{Dovepress}

across all disease areas. The manuscript management system is completely online and includes a very quick and fair peer-review system, which is all easy to use. Visit http://www.dovepress.com/ testimonials.php to read real quotes from published authors. 